\title{
Self-assembly Polyrotaxanes Nanoparticles as Carriers for Anticancer Drug Methotrexate Delivery
}

Longgui Zhang, Ting Su, Bin He, Zhongwei Gu*

Received 27 January 2014; accepted 18 February 2014; published online 20 March 2014)

\begin{abstract}
Cyclodextrin/poly(ethylene glycol) ( $\alpha$-CD/PEG) polyrotaxane nanoparticles were prepared via a self-assembly method. Anticancer drug methotrexate (MTX) was loaded in the nanoparticles. The interaction between MTX and polyrotaxane was investigated. The formation, morphology, drug release and in vitro anticancer activity of the MTX loaded polyrotaxane nanoparticles were studied. The results show that the MTX could be efficiently absorbed on the nanoparticles, and hydrogen bonds were formed between MTX and $\alpha$-CDs. The typical channel-type stacking assembly style of polyrotaxane nanoparticles was changed after MTX was loaded. The mean diameter of drug loaded polyrotaxane nanoparticles were around $200 \mathrm{~nm}$ and the drug loading content was as high as about $20 \%$. Drug release profiles show that most of the loaded MTX was released within 8 hours and the cumulated release rate was as high as 98\%. The blank polyrotaxane nanoparticles were nontoxicity to cells. The in vitro anticancer activity of the MTX loaded polyrotaxane nanoparticles was higher than that of free MTX.
\end{abstract}

Keywords: Polyrotaxane nanoparticles; Drug delivery; Methotrexate; Anticancer activity

Citation: Longgui Zhang, Ting Su, Bin He and Zhongwei Gu, "Self-assembly Polyrotaxanes Nanoparticles as Carriers for Anticancer Drug Methotrexate Delivery", Nano-Micro Lett. 6(2), 108-115 (2014). http://dx. doi.org/10.5101/nml.v6i2.p108-115

\section{Introduction}

Polyrotaxanes have supramolecular architectures with cyclodextrins (CDs) threaded in poly(ethylene glycol) (PEG) chains, they are attractive materials to biomaterials scientists and chemists [1-5]. As CDs and PEG have been approved by Food \& Drug Administration (FDA) in pharmaceutical formulations, the polyrotaxanes exhibit great potential applications in drug delivery. The excellent biocompatibility, multiple functionality and specific self-assembly capabilities are the advantages of polyrotaxanes as drug carriers.

Drug-carriers conjugate is an important form for polyrotaxanes to deliver drugs. With the modification of hydroxyl groups on the CDs, anticancer drugs could be immobilized on polyrotaxanes via cleavable bonds to form drug-polyrotaxane conjugates [6,7]. The drug release was triggered by the broken of the cleavable bonds. High drug loading content was expected to achieve in the conjugates due to the large amount of hydroxyl groups. Targeting moieties such as ligands were also conjugated to polyrotaxanes for multivalent recognization. As CDs could slide along and rotate around the PEG chains, this flexible motion avoided the mismatch of ligand-receptor interaction. This active recognition mode was reported as a new strategy to enhance targeting efficiency $[8,9]$.

Polyrotaxanes were also fabricated non-viral gene vectors. Cationic modified CDs could condense DNA plasmids efficiently $[10,11]$. The sliding and rotation of CDs along the PEG chains provided a "live" style to condense DNA. The N/P ratio of the

National Engineering Research Center for Biomaterials, Sichuan University, Chengdu 610064, China

*Corresponding author. E-mail: zwgu@scu.edu.cn 
DNA/polyrotaxane complex was much lower than that of the DNA/cationic polymer complex under the same DNA condensation condition. Stimuli-sensitive bonds were introduced in the end-caps of polyrotaxane vectors to control the dissociation of the DNA/polyrotaxane complex. The released free DNA was favorable to penetrate into nucleu for efficient transfection. The polyrotaxanes as non-viral gene vectors avoided the over tight condensation of DNA comparing with other non-viral vectors such as cationic polymers.

Polyrotaxane nanoparticles were fabricated via supramolecular self-assembly to deliver anticancer drugs. Early papers have reported polyrotaxane nanoparticles as drug carriers, however, the selfassembly of polyrotaxane nanoparticles were driven by the crystallization of polyrotaxanes [12-14], whose compact architectures hindered the loading of anticancer drugs. In our previous work, we reported a new strategy, small $\pi-\pi$ conjugated molecules such as coumarin derivative and cinnamic acid were immobilized on the terminal groups of PEG chains to prepare amphiphiles $[15,16], \alpha$-cyclodextrins ( $\alpha$-CDs) were threaded in the amphiphiles and the polyrotaxanes self-assembled into nanoparticles. Anticancer doxorubicin was loaded in the nanoparticles via hydrophobic group as well as $\pi-\pi$ stacking interactions between drugs and the end-cap moieties.

Methotrexate (MTX) is an antimetabolite and antifolate drug. It inhibits the metabolism of folic acid for cancer treatment. Herein, we tried to explore the loading of MTX in polyrotaxane nanoparticles. The interaction between MTX and polyrotaxane was investigated. The formation, morphology, drug release and in vitro anticancer activity of the MTX loaded polyrotaxane nanoparticles were studied.

\section{Materials and methods}

\section{Materials}

PEG $(\mathrm{Mn}=2000)$, cinnamic acid and $\alpha$-CD were purchased from Aldrich and vacuum dried at room temperature for $24 \mathrm{~h}$ before used. N,N'dicyclohexylcarbodiimide (DCC), MTX and DMSOd6 were purchased from Aldrich and used as received. Dulbecco's modified Eagle's medium (DMEM), 100× mycillin, and fetal bovine serum (FBS) were used to culture cells for cytotoxicity test. Other chemicals were purchased from Kelong Chemical Co. (Chengdu, China) and used without further purification.

\section{Measurements}

Proton nuclear magnetic resonance $\left({ }^{1} \mathrm{H}\right.$ NMR $)$ spectra were performed on Brukerav- 400 spectrometer with $\mathrm{DMSO}_{d 6}$ as solvents. Tetramethylsilane (TMS) was used as the internal reference. Fourier transform infrared (FT-IR) spectra were recorded on a Thermo Scientific Nicolet iS10 spectrophotometer. Ultrasound was performed on Ultrasonic purifier KQ-300DE, the frequency was at $70 \%$. X-ray diffractometry (XRD) patterns were obtained at room temperature on an X'Pert pro MPD X-ray diffractometer, the powder samples were mounted on a sample holder and scanned from $5^{\circ}$ to $50^{\circ}$. Ultraviolet visible (UV-vis) spectra were recorded on Lambda 650 in a range from $200 \mathrm{~nm}$ to $500 \mathrm{~nm}$. Dynamic light scattering (DLS) experiments were performed on a Malvern ZetasizerNano ZS at an angle of $90^{\circ}$ at $25^{\circ} \mathrm{C}$. Transmission electron microscopy (TEM) was performed on Hitachi H600-4. Atomic force microscopy (AFM) imaging was performed using MFP3D (Asylum Research) with tapping mode.

\section{Synthesis of cinnamic acid modified PEG}

$20 \mathrm{~g}$ of PEG $(\mathrm{Mn}=2000)$ and $4.44 \mathrm{~g}$ of cinnamic acid were dissolved in $200 \mathrm{~mL}$ of dichloromethane, $7.98 \mathrm{~g}$ of DCC dissolved in $100 \mathrm{~mL}$ of dichloromethane was slowly dripped into the mixture in an ice bath. The mixture was stirred in dark at room temperature for 24 $\mathrm{h}$ after the solution of DCC was dripped over. The insoluble N,N-dicyclohexylurea (DCU) was filtered. The filtrate was concentrated in a rotary evaporator. The modified PEG was precipitated in excessive diethyl ether. The product was filtered and dried in vacuum.

\section{Preparation of polyrotaxanes}

$1 \mathrm{~g}$ of cinnamic acid modified PEG was dissolved in $20 \mathrm{~mL}$ of distilled water, the solution was added to 10 $\mathrm{mL}$ of saturated aqueous solution of $\alpha$-CDs. The mixture was ultrasonically agitated for $10 \mathrm{~min}$ and stand at $4^{\circ} \mathrm{C}$ overnight to precipitate white paste. The product was collected by centrifugation, washed with distilled water three times to remove the unthreaded $\alpha$-CDs. The white precipitate was vacuum dried at room temperature to get polyrotaxanes.

\section{Biocompatibility test}

HepG2 cells were cultured in Dulbecco's Modified Eagle's Medium (DMEM) at $37^{\circ} \mathrm{C}$ in $5 \% \mathrm{CO}_{2}$ atmosphere. HepG2 cells harvested in a logarithmic growth phase were seeded on 96-well at a cellular density of $1 \times 10^{4}$ cells/well and incubated for 1 day. Polyrotaxanes were dissolved and diluted in phosphate buffer solution (PBS) buffer with the concentration ranged from $1 \times 10^{-4}$ to $1 \mathrm{mg} / \mathrm{mL}$. The polyrotaxanes and HepG2 cells were incubated for $72 \mathrm{~h}$. The CCK- 8 assay at 490 $\mathrm{nm}$ was performed and the percentage of cell viability was then determined. 


\section{Preparation of MTX loaded polyrotax- ane nanoparticles}

Polyrotaxane $(10 \mathrm{mg})$ and MTX $(5 \mathrm{mg})$ were dissolved in $1 \mathrm{~mL}$ of DMSO. The solution was added into $10 \mathrm{~mL}$ of distilled water dropwise with vigorous stirring in dark. The solution was transferred to a dialysis tubing $(\mathrm{MWCO}=3500)$ and dialyzed at $4^{\circ} \mathrm{C}$ against distilled water for $48 \mathrm{~h}$. The solution in tubing was freezedried to obtain MTX loaded polyrotaxane nanoparticles.

The MTX loaded polyrotaxane nanoparticles were dissolved in $3 \mathrm{~mL}$ of DMSO to determine the drug loading content. The MTX concentration was tested using the UV-vis spectrophotometer at $388 \mathrm{~nm}$. The drug loading content was calculated based on the standard curve obtained from MTX in DMSO. The drug loading content (DLC) and encapsulation efficiency (EE) were calculated according to the following formula:

DLC $($ wt \%) $=$ (weight of loaded drug/weight of drug loaded nanoparticles) $\times 100 \%$

$\mathrm{EE}(\%)=($ weight of loaded drug/weight of drug in feeding $) \times 100 \%$

\section{In vitro drug release}

The drug loaded polyrotaxane nanoparticles were dispersed and diluted in PBS solution to get final concentration of $2 \mathrm{mg} / \mathrm{mL}$. The diluted solution $(0.5 \mathrm{~mL})$ was transferred to dialysis tubing $(\mathrm{MWCO}=3500)$. The tubings were then immersed in a flask containing $25 \mathrm{~mL}$ of PBS solution $(\mathrm{pH}=7.4)$, shaking at a speed of 100 $\mathrm{rev} / \mathrm{min}$ at $37^{\circ} \mathrm{C}$. At specific time intervals, $1 \mathrm{~mL}$ of solution was withdrawn from the release medium and replaced with fresh PBS solution.

\section{In vitro anticancer activity}

HepG2 cells at a logarithm phase were seeded on 96well culture dish at a cell density of $1 \times 10^{4}$ cells/well. After incubated for 1 day, free MTX and MTX loaded polyrotaxane nanoparticles were dispersed and diluted in PBS solution with different concentrations (from 0.001 to $1 \mathrm{mg} / \mathrm{mL}$ ) and incubated for $72 \mathrm{~h}$. The culture medium was removed and the wells were rinsed with PBS for three times ( $\mathrm{pH}=7.4)$. DMEM mediums containing $10 \mu \mathrm{L}$ of CCK- 8 solution was added to each well. After the cells were incubated for additional $2 \mathrm{~h}$, the absorbance was measured in a Thermo Varioskan Flash microplate reader at the wavelength of $490 \mathrm{~nm}$.

\section{Results and discussion}

The schematic illustration of the self-assembly of the drug loaded polyrotaxane nanoparticles was presented in Scheme 1. The cinnamic acid modified PEG threaded in the cavity of $\alpha$-CDs to receive polyrotaxanes. The MTX was loaded in the polyrotaxane nanoparticles. Two drug loaded polyrotaxane nanoparticles with different $\alpha$-CD concentrations in PEG chains were prepared.

${ }^{1} \mathrm{H}$ NMR spectrum was used to characterize the formation of polyrotaxane. The protons of $\mathrm{CH}_{2} \mathrm{CH}_{2} \mathrm{O}$ in PEG chain appeared at $\delta=3.5 \mathrm{ppm}$. The assignment of the protons in $\alpha$-CDs in the polyrotaxanes was shown in Fig. 1. The proton signals of the hydroxyl groups in b-OH and c-OH appeared at $\delta=5.52$ and $5.44 \mathrm{ppm}$, respectively. The signal in $\delta=4.8 \mathrm{ppm}$ was attributed to the proton in carbon (a) in the $\alpha$-CD backbone. The peak at $\delta=4.48 \mathrm{ppm}$ was assigned to the proton in $\mathrm{f}$ $\mathrm{OH}$. As the chemical environment of the protons in the $\alpha$-CD backbone was similar, the chemical shifts of the
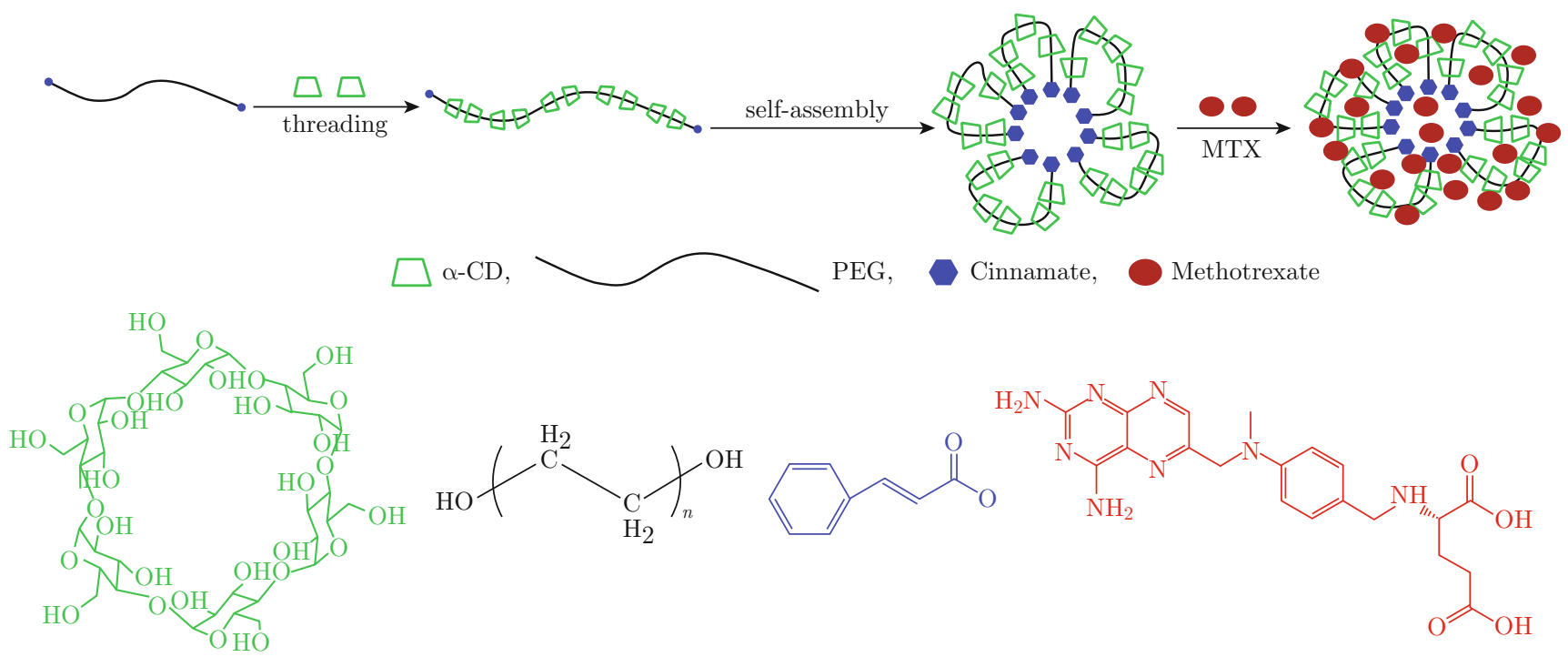

Scheme 1 The illustration of the self-assembly of MTX loaded polyrotaxane nanoparticles. 
protons in the $\alpha$-CD backbone (c, f, e, b and d) were located from $\delta=3.8$ to $3.3 \mathrm{ppm}$, and the signals were split into multiple peaks. The protons in both PEG and $\alpha$-CDs were detected in the spectrum, which revealed that the cinnamic acid modified PEG was threaded in the cavities of $\alpha$-CDs [15], The mole ratio of EG/CD was calculated from the integrities of $\mathrm{OCH}_{2} \mathrm{CH}_{2}$ in $\mathrm{PEG}$ and $\mathrm{CH}(\mathrm{Ca})$ in $\alpha$-CDs. It was reported that the maximum ratio of EG/CD in polyrotaxane was 2.0-2.1 $[17,18]$, The calculated EG/CD ratios of the two polyrotaxanes were 2.9 and 4.3 when the feeding concentrations of $\alpha$-CDs were 1.45 and $0.73 \mathrm{~g} / \mathrm{mL}$ (Table 1 ), which implied that thought the cinnamic acid modified PEG could thread into the cavity of $\alpha$-CDs, the threading capability was weakened by the cinnamate moiety modification.

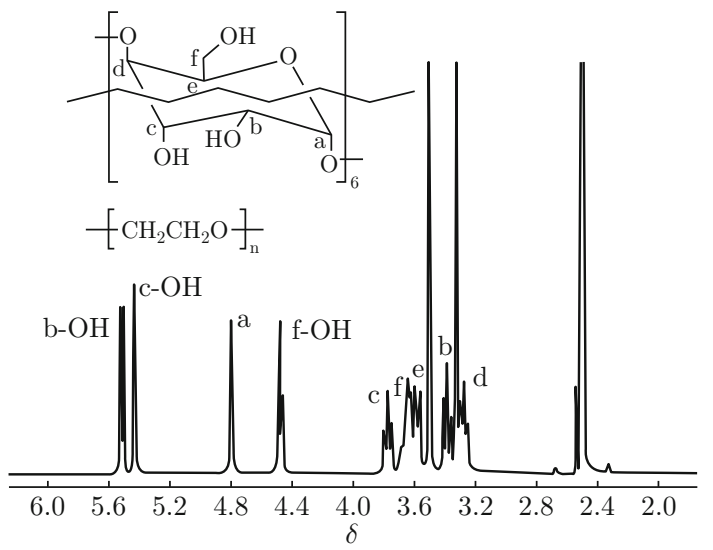

Fig. 1 The ${ }^{1} \mathrm{H}$ NMR spectrum of $\alpha$-CDs/PEG polyrotaxane.

Table 1 The parameters of MTX loaded polyrotaxane nanoparticles

\begin{tabular}{lccccc}
\hline Entry & $\begin{array}{c}\alpha-\mathrm{CD} \\
(\mathrm{g} / \mathrm{mL})^{\mathrm{a}}\end{array}$ & $\begin{array}{c}\text { EG/ } \\
\mathrm{CD}^{\mathrm{b}}\end{array}$ & $\begin{array}{c}\text { DLC } \\
(\%)\end{array}$ & $\begin{array}{c}\mathrm{EE} \\
(\%)\end{array}$ & $\begin{array}{c}\text { Size } \\
(\mathrm{nm})^{\mathrm{c}}\end{array}$ \\
\hline TX/PN-L & 0.73 & 4.3 & 18.7 & 43 & 150 \\
MTX/PN-H & 1.45 & 2.9 & 20.8 & 57 & 140 \\
\hline PN: polyrotaxane nanoparticle. & & & \\
a The concentration in feeding. \\
b Calculated from ${ }^{1} \mathrm{H}$ NMR spectra. \\
c Tested by DLS.
\end{tabular}

The drug loading contents (DLC), encapsulation efficiencies (EE) and mean diameters of the two drug loaded polyrotaxane nanoparticles were summarized in Table 1. The DLC and EE of MTX/PN-H nanoparticles were $20.8 \%$ and $57 \%$, respectively, which were higher than those of MTX/PN-L. These results indicated that the MTX loaded polyrotaxane nanoparticles with high $\alpha$-CDs concentration (MTX/PN-H) exhibited relatively higher DLC and EE. Interestingly, the mean diameter of MTX/PN-H nanoparticles was slightly smaller than that of MTX/PN-L.
In order to explore the interactions between MTX and polyrotaxane nanoparticles, XRD, UV-vis and FTIR spectra of the drug loaded polyrotaxane nanoparticles were measured. XRD is a powerful tool to study the crystal structure of materials. The XRD spectra of blank polyrotaxane nanoparticles, MTX and MTX loaded polyrotaxane nanoparticles were presented in Fig. 2. In the XRD spectrum of blank polyrotaxane nanoparticles, a very strong peak at $2 \theta=19.7^{\circ}$ was presented in the diffraction patterns. It was the characteristic peak associated with a channel-type crystalline structure by virtue of the polymeric nature of the guest molecules [19]. The XRD spectrum demonstrated that the cinnamate moiety modified PEG was threaded in $\alpha$-CDs to form necklace-like polyrotaxanes, which was consistent with the result of ${ }^{1} \mathrm{H}$ NMR spectrum. Free MTX showed strong crystallization capability in the XRD spectrum. However, in the spectrum of MTX loaded polyrotaxane nanoparticles, no strong crystal peaks were observed, it was likely amorphous material, the weak peaks located in the sites of neither polyrotaxane nor MTX. It clearly displayed that the loaded MTX was in amorphous state and it destroyed the crystal structure of polyrotaxane nanoparticles.

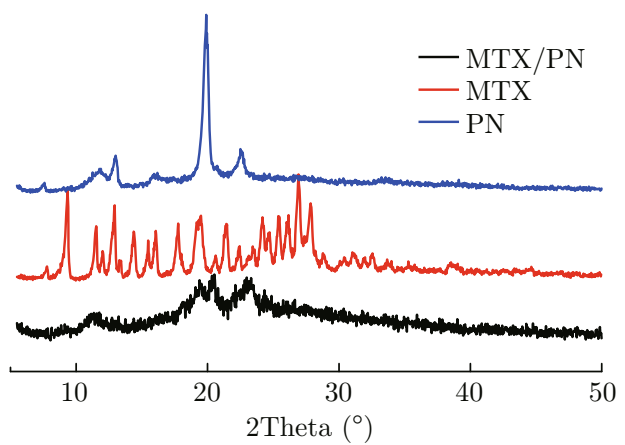

Fig. 2 The XRD spectra of blank polyrotaxane nanoparticles, MTX and MTX loaded polyrotaxane nanoparticles.

The MTX loaded polyrotaxane nanoparticles were dispersed in distilled water to test their UV absorbance (Fig. 3(a)). The spectra of MTX and blank nanopartciles were used as controls. As the strong absorbance of water was below $250 \mathrm{~nm}$, the peaks above $250 \mathrm{~nm}$ were the absorbance of materials. There were two peaks in the UV spectrum of MTX, a strong peak at $302 \mathrm{~nm}$ and a weak shoulder at $350 \mathrm{~nm}$. The UV absorbance peak of blank polyrotaxane nanoparticles appeared at around $280 \mathrm{~nm}$. Comparing with the spectra of MTX loaded polyrotaxane nanoparticles and MTX, it could be found that all the peaks of MTX had red shifts. The peak at $302 \mathrm{~nm}$ was shifted to $306 \mathrm{~nm}$ and the absorbance at $350 \mathrm{~nm}$ was shifted to $378 \mathrm{~nm}$. This red shift revealed that interactions between MTX and polyrotaxane nanoparticles were formed. The UV absorbance of MTX loaded polyrotaxane nanoparticles 
with different concentrations were tested, difference absorbance strength was observed and no red shift was found (Fig. 3(b)). It implied that the red shift was due to the interaction between MTX and polyrotaxane nanoparticles $[20,21]$.

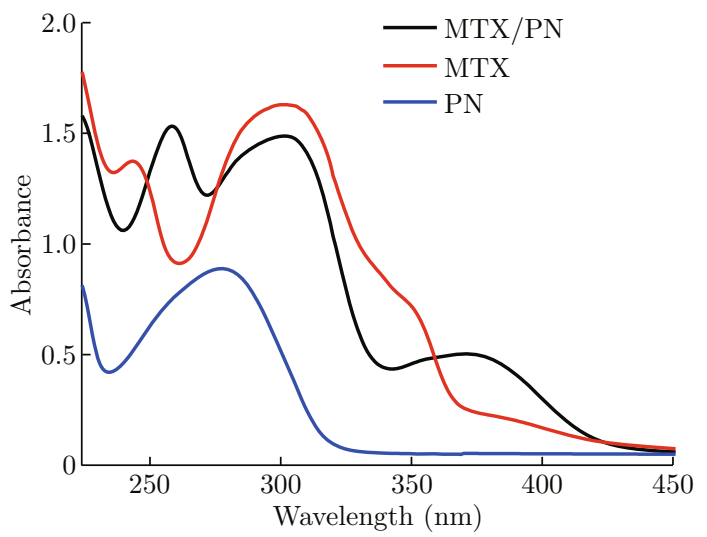

(a)

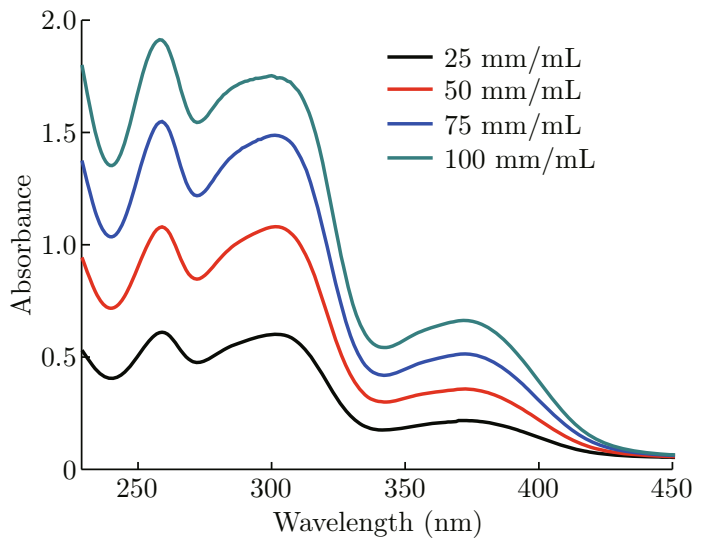

(b)

Fig. 3 The UV spectra of polyrotaxane nanoparticles, MTX and MTX loaded polyrotaxane nanoparticles (a); and MTX loaded polyrotaxane nanoparticles with different concentrations (b).

The FT-IR spectra of MTX, polyrotaxane nanoparticles and MTX loaded polyrotaxane nanoparticles were presented in Fig. 4. Strong vibrations were observed around 3200 to $3600 \mathrm{~cm}^{-1}$, which were attributed to the stretching vibration of hydroxyl and amino groups. The vibration peaks of polyrotaxane nanoparticles, MTX and MTX loaded polyrotaxane nanoparticles were at 3410,3439 and $3423 \mathrm{~cm}^{-1}$, respectively. After MTX was loaded in polyrotaxane nanoparticles, the vibration of MTX was shifted to low wavenumber region, and the vibration of hydroxyl in polyrotaxanes was shifted to higher wavenumber region, which demonstrated the formation of hydrogen bonding [22].

The morphologies of both blank and MTX loaded polyrotaxane nanoparticles were studied by AFM, the images were shown in Fig. 5. In the blank nanoparticle image, regular spherical nanoparticles were observed, the nanoparticles dispersed well in the image and the diameters of the blank $\mathrm{PN}-\mathrm{H}$ polyrotaxane nanoparticles ranged from 100 to $200 \mathrm{~nm}$. The height of the blank nanoparticles measured by AFM was around 10 nm. After MTX was loaded, the shape of the nanoparticles was changed, it was no longer spherical but spindle. The MTX loaded polyrotaxane nanoparticles dispersed well on the mica substrate, the height of MTX loaded polyrotaxane nanoparticles was still about $10 \mathrm{~nm}$. The loading of MTX resulted in the shape change.

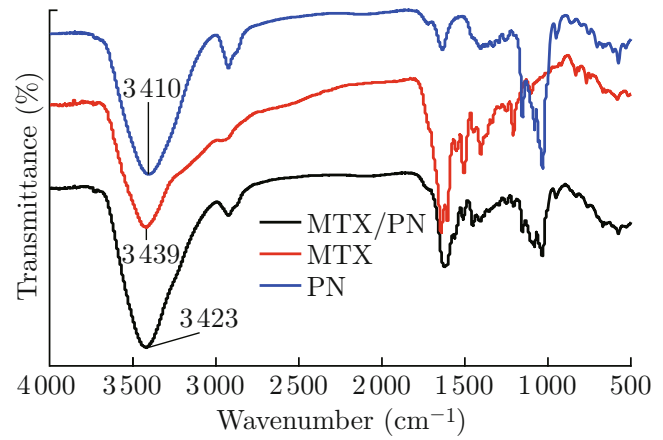

Fig. 4 The FT-IR of blank polyrotaxane nanoparticles, MTX and MTX loaded polyrotaxane nanoparticles.

Two drug loaded polyrotaxane nanoparticles of MTX/PN-L and MTX/PN-H were used to investigate the release profiles (Fig. 6). They were similar, most of the loaded MTX was released within the first four hours, and the cumulated release rates of MTX/PN$\mathrm{L}$ and MTX/PN-H nanoparticles were as high as $98 \%$ and $96 \%$, respectively. The release of $\mathrm{MTX} / \mathrm{PN}-\mathrm{H}$ nanoparticles was a little slower than that of MTX/PN$\mathrm{L}$ nanoparticles. Comparing to other anticancer drug loaded polymeric nanoparticles reported previously [2327], the release of MTX loaded polyrotaxanes nanoparticles was very fast. There were two reasons to explain this phenomenon, one was that the hydrophilicity of MTX was better than other anticancer drugs such as doxorubicin and 9-nitro-20(S)-camptothecin, which resulted faster diffusion from the nanoparticles to themedium. The other reason was that the hydrophobic moieties of the polyrotaxane nanoparticles could not provide enough hydrophobic interaction to encapsulate the MTX inside the nanoparticles, most of the drug was absorbed on the surface of polyrotaxane nanoparticles via Van der Waals' force and hydrogen bonding interaction. Thus, the MTX was released very fast from the polyrotaxane nanoparticles. In cancer therapy, sustaining low dose of anticancer drug was not preferable for cancer treatment, which could not kill cancer cells efficiently and induced multidrug resistance in cancer cells. The polyrotaxane nanoparticles could trigger fast release of MTX, which was potentially favorable for the cancer treatment. 

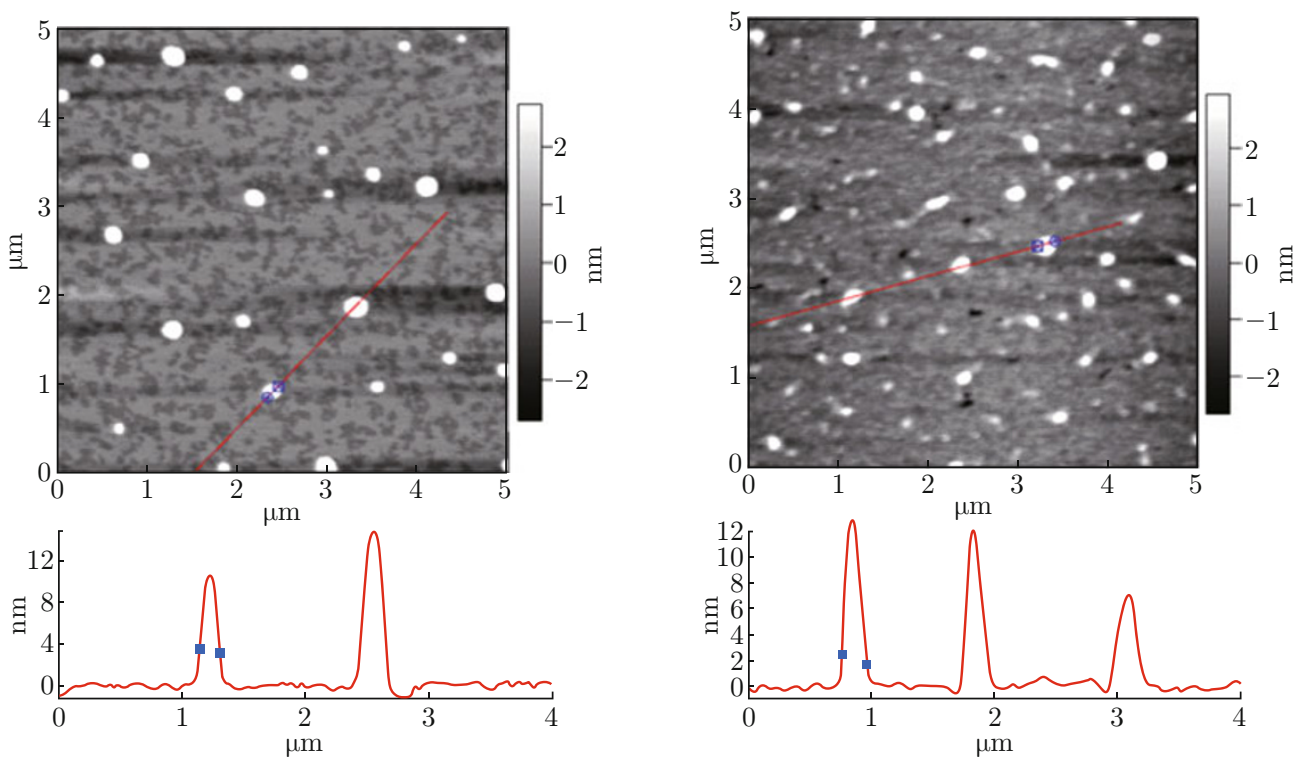

(a)

(b)

Fig. 5 The morphologies of blank polyrotaxane nanoparticles (a) and MTX loaded polyrotaxane nanoparticles (b), the samples were $\mathrm{PN}-\mathrm{H}$ and $\mathrm{MTX} / \mathrm{PN}-\mathrm{H}$.

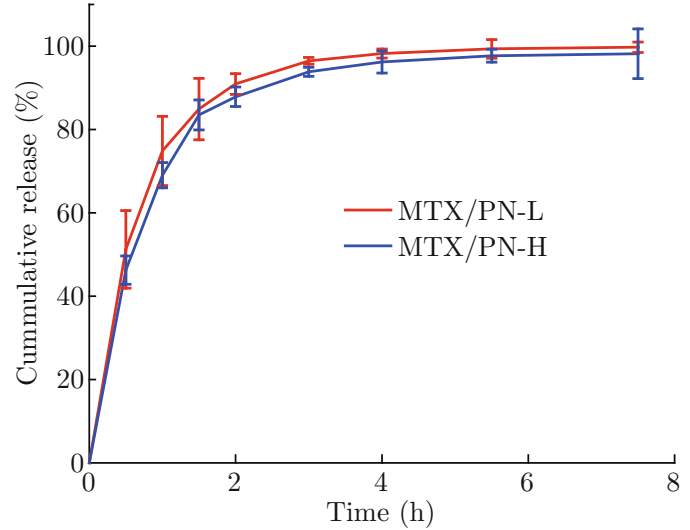

Fig. 6 Drug release profiles of MTX/PN-L and MTX/PNH nanoparticles.

The two blank PN-L and PN-H nanoparticles were incubated with HepG2 cells to evaluate their cytotoxicity. The concentrations of the two nanoparticles were ranged from $1 \times 10^{-4}$ to $1 \mathrm{mg} / \mathrm{mL}$. The cell viabilities of HepG2 cells were presented in Fig. 7 . All the cell viability of the samples were higher than $90 \%$ even when the concentration of nanoparticles was as high as $1 \mathrm{mg} / \mathrm{mL}$, which was much higher than the concentration for anticancer activity study. The cell viability results revealed that the blank polyrotaxane nanoparticles were non-toxic to HepG2 cells.

The in vitro anticancer activity of MTX loaded PN$\mathrm{L}$ and PN-H nanoparticles was tested by CCK- 8 assay. The drug loaded polyrotaxane nanoparticles were incubated with HepG2 cells for $72 \mathrm{~h}$ to evaluate their in vitro anticancer activity. The result showed that MTX loaded PN-L and PN-H nanoparticles killed HepG2

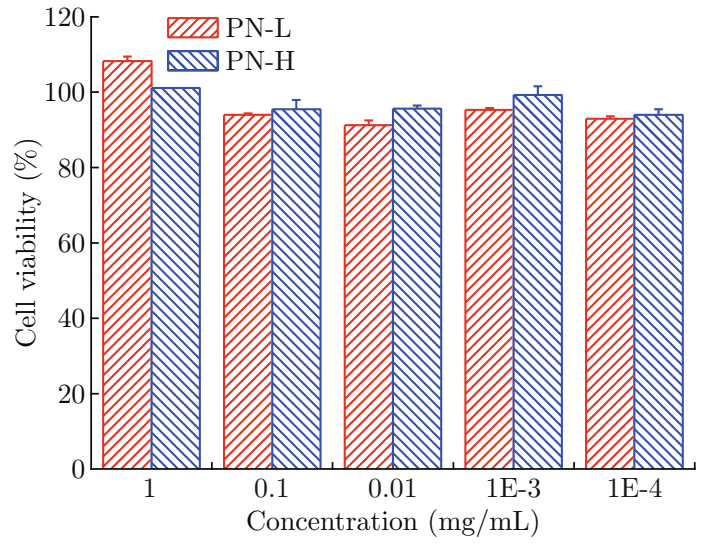

Fig. 7 The cytotoxicity of PN-L and PN-H polyrotaxane nanoparticles.

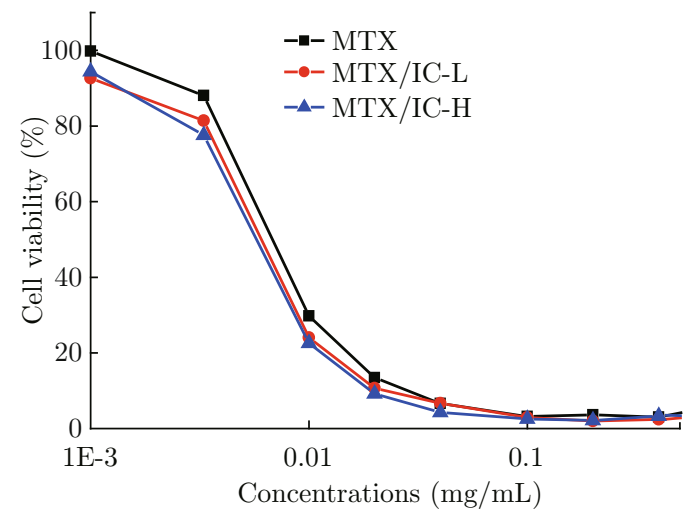

Fig. 8 In vitro anticancer activity of MTX loaded PN-L and $\mathrm{PN}-\mathrm{H}$ nanoparticles against HepG2 cells, the incubation time was 72 hours.

cells in a dose-dependent manner. The value of half maximal (50\%) inhibitory concentration (IC50) of 
MTX loaded PN-L and PN-H nanoparticles calculated from the inhibition cures in Fig. 8 were 6.1 and 5.5 $\mathrm{ng} / \mathrm{mL}$, and that of free MTX was $7.0 \mathrm{ng} / \mathrm{mL}$, the lower IC50 represented better anticancer activity. These results indicated that the HepG2 cells were more sensitive to MTX loaded PN-H nanoparticles. The in vitro anticancer activities of the two nanoparticles were higher than that of free MTX, which implied that the in vitro anticancer activity of MTX was enhanced after loaded in polyrotaxane nanoparticles.

\section{Conclusions}

$\alpha$-CD/PEGpolyrotaxane nanoparticles were fabricated to load anticancer drug MTX. The size of drug loaded nanoparticles was around $150 \mathrm{~nm}$ and the drug loading content was around $20 \%$. The MTX was absorbed on the polyrotaxane nanoparticles and hydrogen bonding between MTX and polyrotaxane nanoparticles was formed. The loaded MTX destroyed the crystal of polyrotaxanes and the UV absorbance peaks of MTX loaded polyrotaxane nanoparticles exhibited red shift. The MTX loaded polyrotaxane nanoparticles were in spindle shape, which were different from regular spherical shape to blank polyrotaxane nanoparticles. The loaded MTX was rapidly released from polyrotaxane nanoparticles and the cumulated release rate was as high as $98 \%$ within the first four hours. The polyrotaxane nanoparticles were non-toxic to HepG2 cells and the anticancer activity of MTX loaded polyrotaxane nanoparticles was better than that of free MTX.

\section{Acknowledgements}

This research was supported by National Science Foundation for Excellent Young Scholars (No. 51222304), National Science Foundation of China (NSFC, No. 31170921, 51133004), National Basic Research Program of China (National 973 program, No. 2011CB606206), and program for Changjiang Scholars and Innovative Research Team in University (IRT1163).

\section{References}

[1] C. C. Rusa, T. A. Bullions, J. Fox, F. E. Porbeni, X. Wang and A. E. Tonelli, "Inclusion compound formation with a new columnar cyclodextrin host", Langmuir 18(25), 10016-10023 (2002). http:// dx.doi.org/10.1021/la0262452

[2] I. N. Topchieva, A. E. Tonelli, I. G. Panova, E. V. Matuchina, F. A. Kalashnikov, V. I. Gerasimov, C. C. Rusa, M. Rusa and M. A. Hunt, "Two-phase channel structures based on $\gamma$-cyclodextrin-polyethylene gly- col inclusion complexes", Langmuir 20(21), 9036-9043 (2004). http://dx.doi.org/10.1021/la048970d

[3] A. Harada and M. Kamachi, "Complex formation between poly(ethylene glycol) and $\alpha$-cyclodextrin", Macromolecules 23(10), 2821-2823 (1990). http://dx. doi.org/10.1021/ma00212a039

[4] J. Li, X. Ni, Z. Zhou and K. W. Leong, "Preparation and characterization of polypseudorotaxanes based on block-selected inclusion complexation between poly(propylene oxide)-poly(ethylene oxide)poly(propylene oxide) triblock copolymers and $\alpha$ cyclodextrin", J. Am. Chem. Soc. 125(7), 1788-1795 (2003). http://dx.doi.org/10.1021/ja026623p

[5] J. J. Li, F. Zhao and J. Li, "Polyrotaxanes for applications in life science and biotechnology", Appl. Microbiol. Biotechnol. 90(2), 427-443 (2011). http://dx. doi.org/10.1007/s00253-010-3037-x

[6] C. Moon, Y. M. Kwon, W. K. Lee, Y. J. Park and V. C. Yang, "In vitro assessment of a novel polyrotaxane-based drug delivery system integrated with a cell-penetrating peptide", J. Controlled Release 124(1-2), 43-50 (2007). http://dx.doi.org/10.1016/ j.jconrel.2007.08.029

[7] C. Moon, Y. M. Kwon, W. K. Lee, Y. J. Park, L. C. Chang and V. C. Yang, "A novel polyrotaxane-based intracellular delivery system for camptothecin: In vitro feasibility evaluation", J. Biomed. Mater. Res. 84A(1), 238-246 (2008). http://dx.doi.org/10.1002/jbm.a. 31452

[8] N. Yui and T. Ooya, "Molecular mobility of interlocked structures exploiting new functions of advanced biomaterials", Chem. Eur. J. 12(26), 6730-6737 (2006). http://dx.doi.org/10.1002/chem. 200600370

[9] N. Yui, R. Katoono and A. Yamashita, "Functional cyclodextrinpolyrotaxanes for drug delivery", Adv. Polym. Sci. 222, 115-173 (2009). http://dx.doi.org/ 10.1007/12_2008_8

[10] T. Ooya, H. S. Choi, A. Yamashita, N. Yui, Y. Sugaya, A. Kano, A. Maruyama, H. Akita, R. Ito, K. Kogure and H. Harashima, "Biocleavablepolyrotaxaneplasmid DNA polyplex for enhanced gene delivery", J. Am. Chem. Soc. 128(12), 3852-3853 (2006). http:// dx.doi.org/10.1021/ja055868+

[11] J. Li, C. Yang, H. Z. Li, X. Wang, S. H. Goh, J. L. Ding, D. Y. Wang and K. W. Leong, "Cationic supramolecules composed of multiple, oligoethylenimine-grafted beta-cyclodextrins threaded on a polymer, chain for efficient gene delivery", Adv. Mater. 18(22), 2969-2974 (2006). http://dx.doi.org/ 10.1002/adma. 200600812

[12] L. X. Ren, F. Y. Ke, Y. M. Chen, D. H. Liang and J. Huang, "Supramolecular ABA triblock copolymer with polyrotaxane as B block and its hierarchical selfassembly", Macromolecules 41(14), 5295-5300 (2008). http://dx.doi.org/10.1021/ma800632m

[13] J. Huang, L. X. Ren and Y. M. Chen, "pH/temperature-sensitive supramolecular micelles based on cyclodextrinpolyrotaxane",Polym. Int. 57(5),714721 (2008). http://dx.doi.org/10.1002/pi. 2396 
[14] C. C. Tsai, S. Leng, K. Jeong, V. R. M. Horn, C. L. Wang, W. B. Zhang, M. J. Graham, J. Huang, R. M. Ho, Y. Chen, B. Lotz and S. Z. D. Cheng, "Supramolecular structure of $\beta$-cyclodextrin and poly(ethylene oxide)-blockpoly(propylene oxide)-block-poly(ethylene oxide) inclusion complexes", Macromolecules 43(22), 9454-9461 (2010). http://dx.doi.org/10.1021/ma101943k

[15] J. Chang, Y. Li, G. Wang, B. He and Z. W. Gu, "Fabrication of novel coumarin derivative functionalized polypseudorotaxane micelles for drug delivery",Nanoscale 5, 813-820 (2013) http://dx.doi.org/ $10.1039 / \mathrm{c} 2 \mathrm{nr} 32927 \mathrm{a}$

[16] R. Liu, Y. S. Lai, B. He, Y. Li, G. Wang, S. Chang and Z. W. Gu, "Supramolecular nanoparticles generated by the self-assembly of polyrotaxanes for anti-tumor drug delivery", Int. J. Nanomed. 7, 5249-5258 (2012). http://dx.doi.org/10.2147/ijn.s33649

[17] A. Harada, J. Li and M. Kamachi, "The molecular necklace: a rotaxane containing many threaded $\alpha$ cyclodextrins", Nature 356, 325-327 (1992). http:// dx.doi.org/10.1038/356325a0

[18] A, Harada, J. Li and M. Kamachi, "Preparation and properties of inclusion complexes of poly(ethylene glycol) with $\alpha$-cyclodextrin", Macromolecules 26(21), 5698-5703 (1993). http://dx.doi. org/10.1021/ma00073a026

[19] T. Uyar, M. A. Hunt, H. S. Gracz and A. E. Tonelli, "Crystalline cyclodextrin inclusion compounds formed with aromatic guests:guest-dependent stoichiometries and hydration-sensitive crystal stuctures", Cryst. Growth Des. 6(5), 1113-1119 (2006). http:// dx.doi.org/10.1021/cg050500+

[20] Y. Lai, Y. Lei, X. Xu, Y. Li, B. He and Z. Gu, "Polymeric micelles with $\pi-\pi$ conjugated cinnamic acid as lipophilic moieties for doxorubicin delivery", J. Mater.
Chem. B 1, 4289-4296 (2013). http://dx.doi.org/ 10.1039/c3tb20392a

[21] S. Pan, S. Luo, S. Li., Y. Lai, Y. Geng, B. He. and Z. $\mathrm{Gu}$, "Ultrasound accelerated gelation of novel L-lysine based hydrogelators", Chem. Comm. 49, 8045-8047 (2013). http://dx.doi.org/10.1039/c3cc44767g

[22] B. He and M. B. Chan-Park, "Synthesis and charaterization of functionalizable, biodegradable and photopatternablepoly ( $\varepsilon$-caprolactone-co-RS- $\beta$-malic acid)", Macromolecule 38(20), 8227-8234 (2005). http://dx.doi.org/10.1021/ma050545j

[23] Y. Lei, Y. Lai, Y. Li, S. Li, G. Cheng, D. Li, H. Li, B. He and Z. Gu, "Anticancer drug delivery of PEG based micelles with small lipophilic moieties", Int. J. Pharm. 453(2), 579-586 (2013). http://dx.doi.org/ 10.1016/j.ijpharm. 2013.06.001

[24] D. Li, Y. Liang, Y. Lai, G. Wang, B. He and Z. Gu, "Polymeric micelles with small lipophilic moieties for drug delivery",Colloids Surf. B:Biointerface http:// dx. doi.org/10.1016/j.colsurfb. 2013.10.032

[25] B. Xue, Y. Wang, X. Tang, P. Xie, Y. Wang, F. Luo, C. $\mathrm{Wu}$ and Z. Qian, "Biodegradable self-assembled mPEG-PCL micelles for hydrophobic oridonin delivery in vitro",J. Biomed. Nanotechnol. 8(1),80-89 (2012). http://dx.doi.org/10.1166/jbn.2012.1358

[26] M. Gou, K. Men, H. Shi, M. Xiang, J. Zhang, J. Song, J. Long, Y. Wan, F. Luo, X. Zhao and Z. Qian, "Curcumin-loaded biodegradable polymeric micelles for colon cancer therapy in vitro and in vivo", Nanoscale 3,1558-1567 (2011). http://dx.doi.org/ 10.1039/c0nr00758g

[27] X. Deng, X. Xu, Y. Lai, B. He and Z. Gu, "Novel nanoparticles generated by polymeric amphiphiles with $\pi-\pi$ conjugated small molecules for anti-tumor drug delivery", J. Biomed. Nanotechnol. 9(8), 13361344 (2013). http://dx.doi.org/10.1166/jbn. 2013. 1626 